\title{
Application of molecular imaging combined with genetic screening in diagnosing MELAS, diabetes and recurrent pancreatitis
}

\author{
Wang Zhiping ${ }^{1 \#}$, Li Quwen ${ }^{2 \#}$, Zhou Hai ${ }^{1}$, Zhou Jian ${ }^{1}$, Gao Peiyi ${ }^{1}$ \\ ${ }^{1}$ Department of Radiology, Beijing Tian Tan Hospital, Capital Medical University, Beijing, ${ }^{2}$ Department of Priority Laboratory \\ for Zoonoses Research, Fujian Central for Disease and Prevention, Fuzhou, China \\ \#These authors contributed equally to this manuscript.
}

\begin{abstract}
Aim: We report molecular imaging combined with gene diagnosis in a family with 7 members who carried an $A 3243 G$ mutation in mitochondrial tRNA and p.Thr 137 Met in cationic trypsinogen (PRSS1) gene presented with mitochondrial encephalomyopathy, lactic acidosis, and stroke-like episodes (MELAS), diabetes, and recurrent pancreatitis.

Material and methods: DNA sequencing was used to detect and validate mitochondrial DNA and PRSS1. We also verified that mitochondrial heterozygous mutations and c.410 C>T mutation causing p.Thr 137 Met could be detected in oral epithelial cells or in urine sediment cells. In addition, molecular imaging was carried out in the affected family members.

Results: In this pedigree, MELAS syndrome accompanied by pancreatitis was an important clinical feature, followed by diabetes. Heteroplasmy of the mTDNA A3243G and C.410 C>T mutation of PRSS1 was found in all tissue samples of these patients, but no mutations were found in 520 normal control and normal individuals of the family. However, based on molecular imaging observations, patients with relatively higher lactate/pyruvate levels had more typical and more severe symptoms, particularly those of pancreatic disease (diabetes or pancreatitis).

Conclusions: MELAS syndrome may be associated with pancreatitis. For the diagnosis, it is more reasonable to perform molecular imaging combined with gene diagnosis.
\end{abstract}

Key words: MELAS, pancreatitis, PRSS1 mutation, molecular imaging, clinical features.

\section{Introduction}

Mitochondrial myopathy, encephalopathy, lactic acidosis, and stroke-like episodes syndrome (MELAS) are established phenotypes of mitochondrial encephalomyopathy. MELAS has been reported worldwide in recent decades $[10,20,22]$. It has been confirmed that a mitochondrial DNA (mtDNA)
3243A $>G$ transition was associated with MELAS in most of these patients and that mutations in mtDNA could be useful for revealing the nature of MELAS. However, reports of MELAS combined with pancreatitis are uncommon and its pathogenesis remains unclear. In addition, a misdiagnosis or a missed diagnosis of pancreatitis combined with MELAS could be 
fatal $[1,5]$. In this paper, we report on a Chinese family afflicted by MELAS combined with diabetes and recurrent pancreatitis.

MELAS, which forms a major clinical subgroup of the mitochondrial encephalomyopathies, is caused by any of several different single base replacements in the mt tRNA ${ }^{\text {Leu(UUR) }}$ gene, which is responsible for the translation of UUR ( $R=A$ or $G$ ) leucine codons in mitochondrial genes [1-5]. It has been reported that $15 \%$ of patients with mitochondriopathies have symptoms of digestive disorders $[3,7,14]$. In contrast, mitochondrial dysfunction in the pancreas frequently results in diabetes mellitus and exocrine insufficiency, but only rarely leads to acute or chronic pancreatitis. Therefore, it was necessary to study the differences between the patients from this pedigree, particularly with regard to the differential diagnosis of pancreatitis.

In recent years, scientific advances have provided us with more comprehensive and objective insights into the pathophysiology of pancreatitis. Since the first discovery of the trypsinogen gene (PRSS1) mutations in hereditary pancreatitis, a variety of gene defects associated with pancreatitis have been reported. Mutations that lead to the generation of more trypsin, or reduce the activity of trypsin inhibitors or trypsin degradation, have been reported to be associated with pancreatitis, either alone or in epistasis. This has broadened the horizon to understand the mechanisms of the disease, and has helped to identify those who are at risk of developing pancreatitis. For many years, inappropriate intra-acinar trypsinogen activation has prevailed as a key initiator of pancreatitis, although the evidence is not direct or concrete $[13,17]$. Continued experimental studies are necessary to determine the specific relations of trypsin-antitrypsin imbalance and genetic heterogeneity in MELAS.

\section{Material and methods \\ Patients}

This study was approved by the Capital Medical University Committee and Fujian Central for Disease and Prevention Committee and all study participants gave informed consent to DNA analyses. Clinical information for the proband and 4 other family members was obtained by personal interviews using a structured questionnaire and/or clinical trials.

\section{Materials}

Mutant loads from the female carriers were assessed using peripheral blood samples and, if possible, urine and/or oral mucosa samples. In addition, hair follicles and muscle tissue were taken from the proband and his mother.

\section{Clinical data}

A total of 5 members from the maternal pedigree were included in this survey (Fig. 1): 2 males (II3, III1) and 3 females $(12,112,115)$. We recorded age, height, body weight, and other physiological conditions for the maternal pedigree. Any history of seizures and stroke-like episodes were recorded. Subjects took a Mini-Mental State Examination (MMSE) to evaluate their cognitive function. A standard vision chart, and pure tone and audiometry were used to determine vision and hearing conditions. We also tested ECG, EEG, and EMG. All family members also had tests for plasma lactate while in a resting state and at 15 minutes after a stepping exercise.

\section{DNA extraction and molecular genetic analysis}

Genomic DNA was extracted from peripheral blood and other tissue specimens using a QIAamp DNA mini kit (Qiagen, Germany). Three genes involved in pancreatitis - PRSS1, SPINK1, and CFTR - were sequenced according to references $[11,12,18]$. The mtDNA3243 A>G and PRSS1 c.410 C >T mutations (p.Thr 137 Met) were examined by polymerase chain reaction (PCR) and direct sequencing. For sequencing, a Perkin Elmer Big Dye Sequencing kit (Perkin-Elmer,

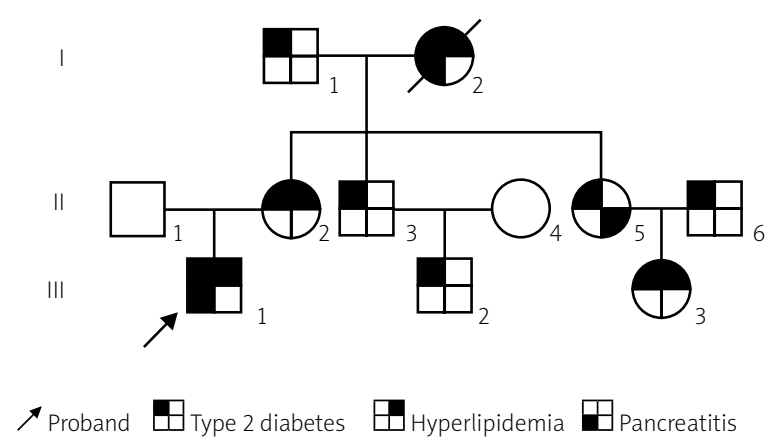

Fig. 1. Pedigree of the family carrying the mitochondrial DNA A3243G and PRSS1 p.Thr 137 Met mutation. 
Shelton, CT, USA) and an ABI PRISM7700 sequencer (Perkin-Elmer ABI, Foster City, CA) were used.

\section{Results}

\section{Clinical data and ancillary test}

Patient III1, a man born in 1992, had non-insulin-dependent (type 2) diabetes mellitus that began at the age of 16 and pancreatitis that first time occurred at the age of 10 . Recent hospitalization was because of paroxysmal unconsciousness, convulsions limbs for 9 days and repeated vomiting for 7 days. The patient was diagnosed with emaciation, memory, computing and understanding power decreased significantly, tendon reflexes slowly and meningeal irritation-positive. CSF pressure was $80 \mathrm{~mm} \mathrm{H} \mathrm{H}_{2}$, lactic acid was $3.5 \mathrm{mmol} / \mathrm{l}$ (normal $<2.1 \mathrm{mmol} / \mathrm{l}$ ) and sugar, protein and $\mathrm{Cl}^{-}$were normal. Plasma lactate $(\mathrm{L})$ was $3.18 \mu \mathrm{mol} / \mathrm{l}$, pyruvate $(\mathrm{P})$ was $0.154 \mu \mathrm{mol} / \mathrm{l}$ and the ratio of $\mathrm{L} / \mathrm{P}$ is 20.60 , serum amylase fluctuations were 258-602 U/l. The proband was given control of epilepsy, gastrointestinal decompression and parenteral nutrition and high doses of coenzyme $Q$ and symptoms were improved after treatment. The man strictly follows doctor's orders and is followed up every 3 months and acquisition of peripheral blood, hair follicle and urine sediment samples from the patients was under the approval of Fujian Medical Ethics Committee.

PCR-RFLP and molecular genetic analysis results are shown in Figure 2 and 3. Heteroplasmy for the A3243G mutation in mtDNA was found in all samples from the affected patients (II2, $\|3\| 5,,\|\| 1)$. The proportion of mutated mtDNA varied from 36 to $73 \%$, depending on the tissue analyzed. In selected patients of non-disease state, there were no significant differences in heteroplasmy for the A3243G mutation in mtDNA. In addition, in the affected patients, no mutations were found in the genes coding for PRSS1, SPINK1, and CFTR.

\section{Molecular genetic analysis}

Heteroplasmy for the A3243G mutation in mtDNA was found in all samples from the affected patients. The proportion of mutated mtDNA varied from 36 to $73 \%$, depending on the tissue analyzed. In selected patients of non-disease state, there were no significant differences heteroplasmy for the A3243G mutation in mtDNA. In addition, in the affected patients, no mutations were found in the genes coding for PRSS1, SPINK1, and CFTR.
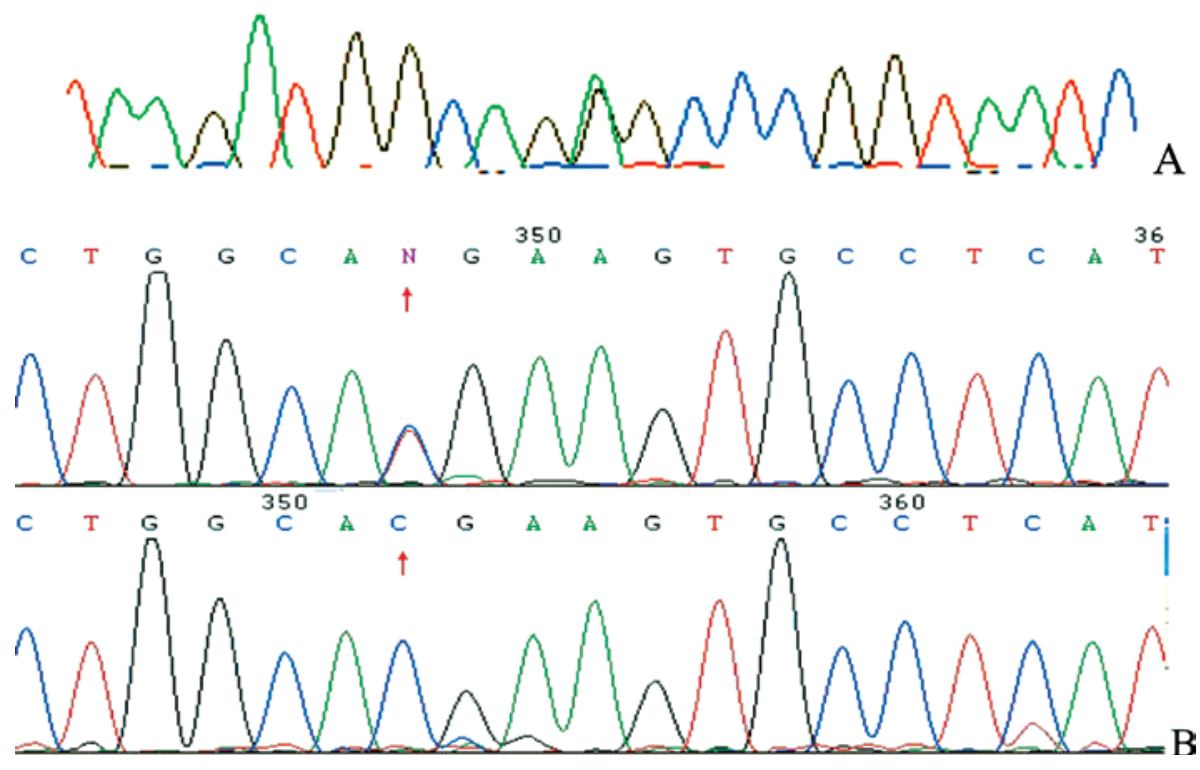

Fig. 2. Mutations of mtDNA and the PRSS1 gene. A) A3243G mutation in mtDNA. B) c.410 C>T (p.T137M) mutation in the PRSS1 gene. 
A
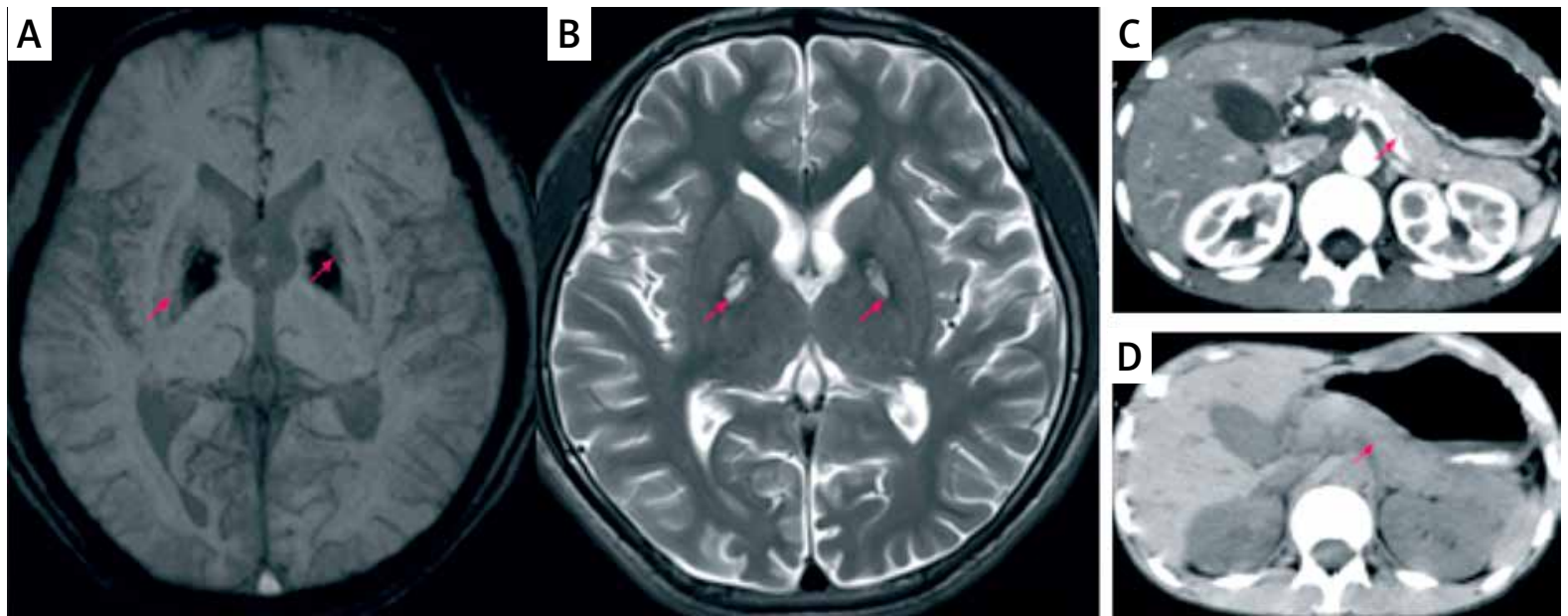

\begin{tabular}{|c|c|c|c|}
\hline G Metabolite & mos./ppm & integral & Ratio \\
\hline Man & 2.02 & 11.21 & 1.97 \\
Cr & 3.04 & 5.70 & 1.00 \\
Cho & 3.22 & 5.76 & 1.01 \\
Crz & 3.03 & 5.00 & 0.08 \\
\hline
\end{tabular}
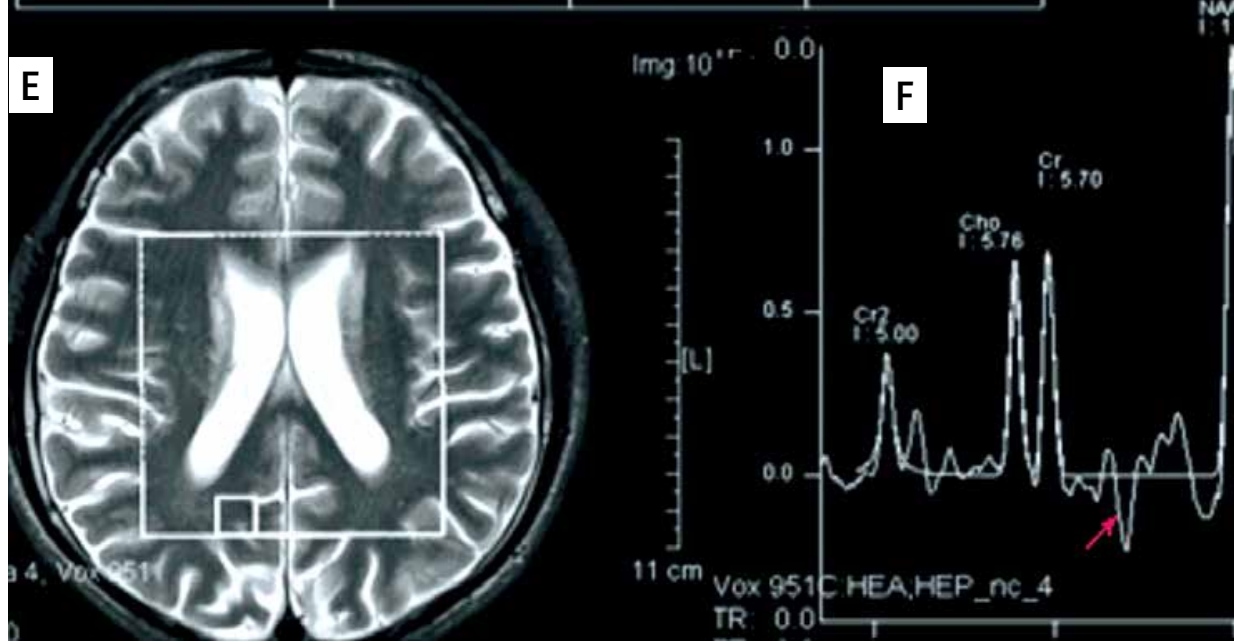

ming

AFF 1 Hos

0001525

Fig. 3. Proband imaging findings. A) and B) Brain MRI findings. C) Enhanced CT findings of pancreas.

D) CT findings of pancreas. E-G) Molecular imaging findings.

\section{Imaging data}

Imaging showed bilateral malacia limb internal capsule, surrounding hemosiderin deposition, symmetry of abnormal signal intensity in the bilateral caudate nucleus, brain atrophy, an inverted lactate peak could be seen in bilateral basal ganglia, bilateral dorsal thalamus and parietal white matter, and the NAA/Cho ratio was not reduced suggesting the local accumulation of lactic acid. His pancreas was fully formed and had a small amount of leakage around it. It had a dilated descending part, considered to be due to an obstruction.

\section{Discussion}

The $A 3243 G$ transition in the MTTL 1 mitochondrial gene is the most common pathogenetic mutation found in mtDNA $[2,11,12,18]$. This mutation is associated with a broad spectrum of clinical manifestations, including mitochondrial encephalomyopathy, lactic acidosis, stroke-like episodic syndrome (MELAS) and maternally inherited diabetes and deafness syndrome (MIDD) $[4,8,15,21]$. Diabetes mellitus and exocrine insufficiency are the most common pancreatic features of mitochondriopathy. In contrast, to date there have been only six reports of patients with 
acute or chronic pancreatitis associated with mtDNA mutations. Four of these cases involved the A3243G mutation. Therefore, clinicians should be aware of the possibility of a missed diagnosis or a misdiagnosis of MELAS accompanied by pancreatitis [9]. The molecular diagnostic accuracy of MELAS is directly related to the choice of samples as the mitochondrial mutation load is quite different in different tissues and the mutation load is usually low in peripheral blood [16]. This is because mitochondrial DNA is divided randomly among daughter cells during mitosis and the daughter cells have different proportions of mutant or wild-type mitochondrial DNA.

In this study, urinary sediment cells and oral epithelial cells were collected, which have good diagnostic performance and are convenient samples to be collected for non-invasive screening of the family members of patients. The hair follicle is also a sensitive tissue that has the $A 3243 G$ point mutation in its mitochondrial DNA. During aging, this mutation will disappear in blood cells, while hair follicles will maintain this mutation and have the advantages of ease of collection and storage, and can also be used to screen asymptomatic family members for genetic counseling.

The diagnosis and differential diagnosis of MELAS should be based on clinical symptoms, molecular biology, iconography, and other comprehensive analyses. The standard for detection is the sequencing of nuclear gene mutations. However, in the field of molecular diagnostics for mitochondrial diseases, owing to the considerably high frequency of mtDNA polymorphisms, which often is the variation due to homogeneity, and the limitations of sequencing, it is considered that those cases with mutation rates of $0-20 \%$ or $80-100 \%$ can easily be mistaken as homogeneity. Therefore, for the screening of heterogeneous cases, the sequencing method should be used in conjunction with other methods, such as restriction enzyme digestion, PCR primer mismatch, and other methods that can, to some extent, compensate for the disadvantages of sequencing for predicating possible heterozygous mutations. In addition, the proportions of different genotypes can be obtained when using gel scanning technology.

In the clinical diagnosis of mitochondrial disease, because of a variety of internal and external factors, the functional status of mitochondria is enormously diverse and it is difficult to confirm a precise definition of encephalomyopathy from any single aspect of clinical symptoms, pathological or biochemical. MtDNA mutation testing is helpful for diagnosing mitochondrial diseases, but it is not the only means. In recent years, with the rapid development of molecular imaging technology (spectroscopy), the direct finding of a lactate peak (areas of lactic acid accumulation) in the pineal body region using computer integration of data has contributed to the diagnosis of MELAS [19].

Pancreatitis may also be explained by a similar mitochondrial energy defect or vascular dysfunction. Although there is no direct evidence for vascular involvement in the pancreas of patients with the $A 3243 G$ mutation, it is known that this organ is susceptible to ischemic injury, and perturbations of the systemic and pancreatic micro-vascularization play a significant role in the pathogenesis of pancreatitis [6]. In addition, mitochondrial diseases, including MELAS, are associated with an excess production of reactive oxygen species (ROC), which mostly occurs in the mitochondria as byproducts of oxidative phosphorylation, and has been described as an important factor in the pathogenesis and progression of pancreatitis [23].

In patients with MELAS, non-specific gastrointestinal symptoms, including constipation, stomach upset, liver disease, recurrent vomiting or recurrent pancreatitis, are being increasingly recognized. Different diseases share the same point base mutation leading to an "overlap syndrome" and the same clinical phenotypes can be caused by mutations at multiple sites. This means that it remains unknown whether the clinical symptoms of mitochondrial diseases are caused by a single gene mutation or by the interaction of mutations at different sites and heterogeneity. Thus, there are many problems that remain to be explored.

\section{Disclosure}

Authors report no conflict of interest.

\section{References}

1. Bouchet C, Steffann J, Corcos J, Monnot S, Paquis V, Rötig A, Lebon S, Levy P, Royer G, Giurgea I, Gigarel N, Benachi A, Dumez Y, Munnich A, Bonnefont JP. Prenatal diagnosis of myopathy, encephalopathy, lactic acidosis, and stroke-like syndrome: contribution to understanding mitochondrial DNA segregation during human embryofetal development. J Med Genet 2006; 43: 788-792.

2. Brandon MC, Lott MT, Nguyen KC, Spolim S, Navathe SB, Baldi P, Wallace DC. MITOMAP: a human mitochondrial genome database - 2004 update. Nucleic Acids Res 2005; 33: 611-613. 
3. Burim RV, Canalle R, Martinelli Ade L, Takahashi CS. Polymorphisms in glutathione S-transferases GSTM1, GSTT1 and GSTP1 and cytochromes P450 CYP2E1 and CYP1A1 and susceptibility to cirrhosis or pancreatitis in alcoholics. Mutagenesis 2004; 19: 291-298.

4. Canafoglia L, Franceschetti S, Antozzi C. Epileptic phenotypes associated with mitochondrial disorders. Neurology 2001; 56:1340-1346.

5. Craven L, Tuppen HA, Greggains GD, Harbottle SJ, Murphy JL, Cree LM, Murdoch AP, Chinnery PF, Taylor RW, Lightowlers RN, Herbert M, Turnbull DM. Pronuclear transfer in human embryos to prevent transmission of mitochondrial DNA disease. Nature 2010; 465: 82-85.

6. Cuthbertson CM, Christophi C. Disturbances of the microcirculation in acute pancreatitis. Br J Surg 2006; 93: 518-530.

7. Debray FG, Drouin E, Herzog D, Lortie A, Lambert M, Garel L, Mitchell GA, Michaud JL. Recurrent pancreatitis in mitochondrial cytopathy. Am J Med Genet A 2006; 140: 2330-2335.

8. de Vries MC, Rodenburg RJ, Morava E, van Kaauwen EP, ter Laak H, Mullaart RA, Snoeck IN, van Hasselt PM, Harding P, van den Heuvel LP, Smeitink JA. Multiple oxidative phosphorylation deficiencies in severe childhood multi-system disorders due to polymerase gamma (POLG1) mutations. Eur J Pediatr 2007; 166: 229-234.

9. DiMauro S, Schon EA. Mitochondrial respiratory-chain diseases. N Engl J Med 2003; 348: 2656-2668.

10. Fragaki K, Procaccio V, Bannwarth S, Serre V, O'Hearn S, Potluri P, Augé G, Casagrande F, Caruba C, Lambert JC, Paquis-Flucklinger V. A neonatal polyvisceral failure linked to a de novo homoplasmic mutation in the mitochondrially encoded cytochrome b gene. Mitochondrion 2009; 9: 346-352.

11. Gao F, Li Y, Wang C, Zhuang Z, Liu QC, Chen J, Hong G, Xu Z. Identification of a Novel Frame-Shift Mutation in PRSS1 Gene in Han Patients with Autoimmune Pancreatitis. Curr Mol Med 2014; 14: 340-348.

12. Gao F, Li YM, Hong GL, Xu ZF, Liu QC, He QL, Lin LQ, Weng SH. PRSS1_p.Leu81Met mutation results in autoimmune pancreatitis. World J Gastroenterol 2013; 19: 3332-3338.

13. Giulivi C, Zhang YF, Omanska-Klusek A, Ross-Inta C, Wong S, Hertz-Picciotto I, Tassone F, Pessah IN. Mitochondrial dysfunction in autism. JAMA 2010; 304: 2389-2396.

14. Hom XB, Lavine JE. Gastrointestinal complications of mitochondrial disease. Mitochondrion 2004; 4: 601-607.

15. lizuka T, Sakai F, Endo M, Suzuki N. Response to sumatriptan in headache of MELAS syndrome. Neurology 2003; 61: 577-578.

16. Kishnani PS, Van Hove JL, Shoffner JS, Kaufman A, Bossen EH, Kahler SG. Acute pancreatitis in an infant with lactic acidosis and a mutation at nucleotide 3243 in the mitochondrial DNA tRNALeu (UUR) gene. Eur J Pediatr 1996; 155: 898-903.

17. Lebre AS, Rio M, Faivre d'Arcier L, Vernerey D, Landrieu P, Slama A, Jardel C, Laforêt P, Rodriguez D, Dorison N, Galanaud D, Chabrol B, Paquis-Flucklinger V, Grévent D, Edvardson S, Steffann J, Funalot B, Villeneuve N, Valayannopoulos V, de Lonlay P, Desguerre I, Brunelle F, Bonnefont JP, Rötig A, Munnich A, Boddaert N. A common pattern of brain MRI imaging in mitochondrial diseases with complex I deficiency. J Med Genet 2011; 48: 16-23.

18. Liu QC, Gao F, Ou QS, Zhuang ZH, Lin SR, Yang B, Cheng ZJ. Novel mutation and polymorphism of PRSS1 gene in the Chinese patients with heredity pancreatitis and chronic pancreatitis. Chin M J 2008; 121: 108-111.

19. Möllers M, Maniura-Weber K, Kiseljakovic E, Bust M, Hayrapetyan A, Jaksch M, Helm M, Wiesner RJ, von Kleist-Retzow JC. A new mechanism for mtDNA pathogenesis: impairment of post-transcriptional maturation leads to severe depletion of mitochondrial tRNASer (UCN) caused by T7512C and G7497A point mutations. Nucleic Acids Res 2005; 33: 5647-5658.

20. Moraes CT, Ciacci F, Bonilla E, Ionasescu V, Schon EA, DiMauro S. A mitochondrial tRNA anticodon swap associated with a muscle disease. Nat Genet 1993; 4: 284-288.

21. Schaefer AM, McFarland R, Blakely EL, He L, Whittaker RG, Taylor RW, Chinnery PF, Turnbull DM. Prevalence of mitochondrial DNA disease in adults. Ann Neurol 2008; 63: 35-39.

22. Suzuki T, Suzuki T, Wada T, Saigo K, Watanabe K. Taurine as a constituent of mitochondrial tRNAs: new insights into the functions of taurine and human mitochondrial diseases. EMBO J 2002; 21: 6581-6589.

23. Tsai K, Wang SS, Chen TS, Kong CW, Chang FY, Lee SD, Lu FJ. Oxidative stress: an important phenomenon with pathogenetic significance in the progression of acute pancreatitis. Gut 1998; 42: 850-855. 